\section{Tyler Chapman, DO}

Assistant Professor of Medicine, Penn State

Health, Milton S. Hershey Medical Center,

Hershey, PA
Elizabeth Sinz, MD, MEd

Professor of Anesthesiology and Perioperative

Medicine, Penn State Health, Milton S. Hershey

Medical Center, Hershey, PA
Brian McGillen, MD, FACP, SFHM

Associate Professor of Medicine, Penn State

Health, Milton S. Hershey Medical Center,

Hershey, PA

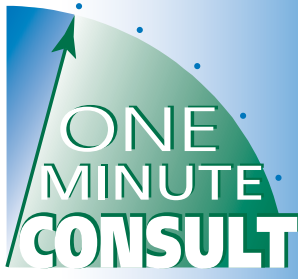

BRIEF ANSWERS

TO SPECIFIC

CLINICAL

QUESTIONS

\title{
: Does my hospitalized patient need an NPO-after-midnight order preoperatively?
}

\begin{abstract}
69-year-old man with osteopenia is brought A to the emergency department after falling while walking on his driveway. He suffered no loss of consciousness. Initial evaluation reveals a right intertrochanteric hip fracture, and he is admitted to the hospital medicine service. On admission, the nocturnist is asked to place an order for nothing by mouth (NPO) after midnight, as the patient will be an "add-on" for surgery the next day. The next day, the patient is taken to the operating room at 3:45 pm, almost 16 hours after the order disallowing food or drink was placed.
\end{abstract}

A: Not every inpatient awaiting surgery under general anesthesia requires an order for nothing by mouth (NPO) after midnight, and it is important for internists to be aware of recommendations on this matter.

Ordering NPO after midnight has been a common practice to lower the risk of aspiration of gastric contents during general anesthesia, which is due in part to suppression of laryngeal reflexes from anesthesia. ${ }^{1,2}$ Unfortunately, delays, cancellations, and postponements of surgical procedures are common, and disallowing oral intake for prolonged periods in these situations may lead to patient harm and frustration. A recent review by Black et $\mathrm{al}^{3}$ reported that orders for NPO after midnight were low-value patient care, a practice that is not evidence-based practice and that causes significant patient dissatisfaction.

Further, the use of midnight as the cutoff for oral intake is arbitrary, chosen more for the convenience of the operating room schedule and the ordering provider rather than the patient. A 2003 Cochrane review ${ }^{4}$ concluded that there was no support for lengthier fasting periods such as the standard NPO after midnight doi:10.3949/ccjm.89a.21061 when compared with shortened fluid fasts. In fact, the American Society of Anesthesiologists (ASA) guidelines for preoperative fasting allow light meals up to 6 hours and clear liquids up to 2 hours before elective procedures utilizing general anesthesia. ${ }^{5}$ These guidelines are similar to those of the European Society of Anaesthesiology and Intensive Care (ESAIC) ${ }^{6}$

\section{CONSIDERATIONS BEFORE ORDERING NPO AFTER MIDNIGHT}

When placing a preoperative NPO order, the following should be considered:

- Orders for NPO after midnight should be used sparingly

- Providers should be aware of the potential harms of prolonged fasting

- When patients are expected to undergo inpatient procedures, communication between providers is key to ensure preoperative recommendations from all teams are followed

- Major society guidelines should serve as a backbone for the development of protocols that utilize dietary modifications and briefer periods of fasting before elective procedures

- Allowing clear liquids 2 hours or a light breakfast 6 hours before administration of anesthesia is preferred.

\section{ELECTIVE OR SEMI-URGENT PROCEDURES}

We suggest that institutions utilize published guidelines as a basis to implement multidisciplinary protocols incorporating brief, evidencebased fasting periods before elective surgical procedures. Furthermore, given the potential harm to the patient of prolonged fasting, an individualized approach should be made regarding NPO-after-midnight orders, including patients undergoing semi-urgent procedures.
Disallowing oral intake for prolonged periods may lead to patient harm and frustration 
The benefits of a patient-centered approach avoiding prolonged fasting when appropriate is well-cited. In a study of bariatric surgery patients, Nossaman et $\mathrm{al}^{7}$ reported that instituting a policy allowing patients access to water up to 2 hours before surgery resulted in shorter hospital length of stay compared with patients in a post hoc analysis before the policy was instituted. Interestingly, no perioperative aspiration events were reported in either group. ${ }^{7}$

\section{PATIENTS AT HIGH RISK OF ASPIRATION}

Prolonged fasting became a standard in the mid-1900s, when catastrophic surgical complications were reported in obstetric patients who aspirated during labor. ${ }^{8}$ Although this practice has incorrectly been extrapolated to nearly all surgical and radiologic procedures, patients in labor, patients with obesity, those with a history of esophageal surgery, and those with gastrointestinal obstruction are at highest risk of aspiration ${ }^{1}$ and thus may require a prolonged fast. Clinicians should be able to identify these patients early in the hospital course when taking a careful history.

\section{THE BOTTOM LINE}

Although the practice of prolonged preoperative fasting has been considered the best way to prevent aspiration, overutilization of NPO-after-midnight orders represents low-value care, and it violates at least 3 of the 6 healthcare quality domains (safety, efficacy, and patientcenteredness) without leading to improved outcomes. Furthermore, prolonged fasting is an obstacle to maintaining appropriate hydration preoperatively, thus making it more difficult to optimize hemodynamics after induction of general anesthesia. ${ }^{9}$ Unfortunately, the evidencebased guidelines for perioperative dietary allowance published by the ASA and ESAIC are not widely adopted as standard of care for inpatients in need of nonelective procedures. ${ }^{5,6}$

Evidence shows that more liberal NPO policies do not increase cases of aspiration or surgical case cancellation. ${ }^{10}$ In the case of the patient in the opening scenario, it would be prudent for the nocturnist to order an early breakfast of clear liquids after a discussion with colleagues in orthopedic surgery and anesthesiology. This should not interfere with the patient's surgery, even if the surgery occurs earlier than originally anticipated.

An optimal approach to NPO in hospitalized patients awaiting surgery would be for overnight nursing staff to be able to confirm operating room scheduling before leaving their shift. Scheduling of add-on surgeries should be refined in patient-centered ways, as this is a root cause leading to the practice of ordering NPO after midnight. This would help ensure that patients receive their meals appropriately. This approach would best be developed through systemic solution planning that would include representatives from nursing (operating room and bedside staff), hospital medicine, surgical and procedural specialties, and anesthesiology.

\section{DISCLOSURES}

The authors report no relevant financial relationships which, in the context of their contributions, could be perceived as a potential conflict of interest.

\section{REFERENCES}

1. Nason KS. Acute intraoperative pulmonary aspiration. Thorac Surg Clin 2015; 25(3):301-307. doi:10.1016/j.thorsurg.2015.04.011

2. Merchant RN, Chima N, Ljungqvist $O$, Kok JNJ. Preoperative fasting practices across three anesthesia societies: survey of practitioners. JMIR Perioper Med 2020; 3(1):e15905. doi:10.2196/15905

3. Black MK, Lupa MC, Lemley LW, Dreesen EB, Deaton AM, Wardrop RM 3rd. Things we do for no reason ${ }^{\mathrm{TM}}$ : NPO after midnight. J Hosp Med 2021; 16(6):368-370. doi:10.12788/jhm.3537

4. Brady M, Kinn S, Stuart P. Preoperative fasting for adults to prevent perioperative complications. Cochrane Database Syst Rev 2003; (4):CD004423. doi:10.1002/14651858.CD004423

5. Practice guidelines for preoperative fasting and the use of pharmacologic agents to reduce the risk of pulmonary aspiration: application to healthy patients undergoing elective procedures: an updated report by the American Society of Anesthesiologists Task Force on Preoperative Fasting and the Use of Pharmacologic Agents to Reduce the Risk of Pulmonary Aspiration. Anesthesiology 2017; 126(3): 376-393. doi:10.1097/ALN.0000000000001452

6. Smith I, Kranke P, Murat I, et al. Perioperative fasting in adults and children: guidelines from the European Society of Anaesthesiology. Eur J Anaesthesiol 2011; 28(8):556-569. doi:10.1097/EJA.0b013e3283495ba1

7. Nossaman VE, Richardson WS, Wooldridge JB Jr, Nossaman BD. Duration of Nil Per Os is causal in hospital length of stay following laparoscopic bariatric surgery. Surg Endosc 2017; 31(4):1901-1905. doi:10.1007/s00464-016-5191-4

8. Mendelson CL. The aspiration of stomach contents into the lungs during obstetric anesthesia. Am J Obstet Gynecol 1946; 52:191-205. doi:10.1016/s0002-9378(16)39829-5

9. Li Y, He R, Ying X, Hahn RG. Dehydration, hemodynamics and fluid volume optimization after induction of general anesthesia. Clinics (Sao Paulo) 2014; 69(12):809-816. doi:10.6061/clinics/2014(12)04

10. Murphy GS, Ault ML, Wong HY, Szokol JW. The effect of a new NPO policy on operating room utilization. J Clin Anesth 2000; 12(1):48-51. doi:10.1016/s0952-8180(99)00139-7

Address: Tyler Chapman, DO, Department of Internal Medicine, Penn State Health, Milton S. Hershey Medical Center, 500 University Drive, Hershey, PA 17036; tchapman@pennstatehealth.psu.edu 
In the February 2022 issue, an error appeared in Chapman T, Sinz E, McGillen B. Does my hospitalized patient need an NPO-after-midnight order preoperatively? Cleve Clin J Med 2022; 89(2):69-70. doi:10.3949/ccjm.89a.21061. In the reference list on page 70, reference 3, the citation information was incorrect. The reference should have read as follows: Black MK, Lupa MC, Lemley LW, Dreesen EB, Deaton AM, Wardrop RM 3rd. Things we do for no reason: NPO after midnight. J Hosp Med 2021; 16(6):368-370. doi:10.12788/jhm.3537. This is now correct on ccjm.org. 